\title{
Eficiencia en la productividad desde la perspectiva del cliente interno y externo en las empresas recicladoras del plástico en el departamento de la Guajira-Colombia
}

\section{Efficiency in productivity from the perspective of internal and external customer in the plastics reclying companies in the department of Guajira - Colombia}

Victor José Iguaran Manjarrés

PostDoctor en Ciencias Humanas Universidad del Zulia, Doctor en Ciencias Gerenciales de la Universidad Rafael Belloso Chacín, Maracaibo- Venezuela, Magister en Gerencia de Mercadeo, Especialista en Gerencia de Mercadeo Universidad Jorge Tadeo Lozano, Administrador de Empresas, profesor Asociado de la Universidad de La Guajira. Correo Electrónico viguaran@uniguajira.edu.co
Ledis Esther Campo Rivadeneira Doctora en Ciencias Políticas de la Universidad Rafael Belloso Chacín, Maracaibo- Venezuela, Magister en Ciencias de la Educación Mención Gerencia Educativa de la Universidad Rafael Belloso Chacín, Maracaibo- Venezuela, Especialista en Gerencia social, Trabajadora Social, profesora Auxiliar de la Universidad de La Guajira, adscrito a la cátedra de Intervención profesional en Trabajo Social, y Gerencia social en la Facultad Ciencias Sociales y Humanas Universidad de la Guajira.

Correo Electrónico lcampo@uniguajira.edu.co 


\section{Resumen}

$\mathrm{L}$ a finalidad del presente artículo es analizar la eficiencia en la produc- Palabras clave: tividad, desde la perspectiva del cliente interno y externo, en las empresas recicladoras del plástico en el departamento de la Guajira-Colombia. Se determina primero la repercusión del cliente interno, que es el personal que labora en las empresas recicladoras, el cual se considera el receptor primario de la visión, misión y estrategias formuladas para lograr la satisfacción del cliente externo. En este orden de ideas, cada empleado es un cliente interno conforme recibe su insumo, información, tarea, entre otros; en tanto que al cliente externo, que es el punto vital para cualquier institución, se le conoce también desde el punto de vista del ciclo comercial como grupo objetivo que pasa por consumidor potencial, luego eventual y, por último, en habitual. En este caso, se evidencia que los recursos y logística que poseen los clientes internos para satisfacer las necesidades, deseos y expectativas de su cliente externo expresan un moderado nivel de productividad. A la vez, se generan lineamientos estratégicos de mercadeo para potencializar el proceso productivo de este sector empresarial.

\section{Abstract}

$\mathrm{T}$ he purpose of this paper is to analyze the efficiency in Productivity from the Perspective of Internal and External Clients in Plastic recycling companies in the Department of Guajira - Colombia as a result of a Strategic Product Marketing Management Research as a Productivity Tool for these Companies. It has been determined the impact that the internal customer has, who is also the working staff in the recycling companies and who is considered the primary recipient of the vision, mission and strategies designed to achieve external customer satisfaction. In this order of ideas, each employee becomes an internal client when he receives his input, information, task, among others; and the external customer which is a vital point for any institution, without whom there will be no reason for work; it is also known, from the point of view of the business cycle, as a target group which pass through potential consumers which eventually become regular customers. It is evident that there is a moderate level of productivity with the resources and logistic that have to meet the needs, desires and expectations of your external customer. At the same time, a strategic marketing guideline that potentiates the production process of this business sector is generated.
Eficiencia, cliente interno, cliente externo, productividad, empresas recicladoras.
Keywords:

efficiency, internal customer, External customer, Productivity, Recycling companies. 


\section{INTRODUCCION}

La productividad es una de las principales preocupaciones de los administradores de este siglo, al punto que rebasa la frontera de países poderosos como Estados Unidos y se extiende a muchas otras partes del mundo. Incluso Japón, admirado por sus mejoras de productividad, se interesa en la actualidad por mantener su competitividad en el mercado mundial. En Latinoamérica ello implica medición, que es un paso esencial del proceso de control. La productividad permite determinar al empresario la relación insumo-producto, lo que sirve, a su vez, para evaluar el rendimiento de los talleres, máquinas, equipo de trabajo y empleados. Por ello, Koontz y Weihrich [1] aseguran que la productividad implica la eficiencia en el desempeño individual y organizacional, y que es el logro de los objetivos con la menor cantidad de recursos. De ahí que una organización se considere productiva cuando consigue sus metas transformando paralelamente sus insumos en productos al menor costo, con el fin de conseguir una satisfacción de su cliente interno y externo, respectivamente.

En tal sentido es pertinente determinar la eficiencia en la productividad desde la perspectiva del cliente interno y externo en las empresas recicladoras del plástico en el departamento de La Guajira-Colombia, con la finalidad de que se aproveche en forma óptima la significativa cantidad de plástico reciclable que se genera en la zona fronteriza colombo-venezolana. Lo anterior con el propósito final de planificar la actividad del reciclaje, que en los momentos actuales se realiza informalmente, marcada, además, por el estigma social que soportan quienes trabajan con residuos sólidos, sin efectuar procesos productivos eficientes.

En La Guajira colombiana se generan aproximadamente 1029 toneladas de residuos sólidos por día, de estos el $35 \%$ es plástico, lo que, según el Instituto de Bienestar Familiar Regional Guajira (2015), representa un total de 360 toneladas por día. El 85\% de estos son vertidos en los rellenos sanitarios porque no se realiza un proceso de selección racional, que pueda ser aprovechado como materia prima por las empresas naturales y jurídicas que se dedican a reciclar.

En el departamento de La Guajira, casi dos mil personas se dedican a la actividad del reciclaje del plástico, y acopian ciertas cantidades de este material para ser procesado y comercializado. Pero en su labor presentan un sinnúmero de problemas, como falta de seguridad social e ínfimos niveles culturales y educativos, problema debido a las limitadas posibilidades de los individuos para recoger diariamente suficientes residuos sólidos. Normalmente, quienes se dedican a esta actividad no disponen de equipos necesarios, carecen de lugares para el almacenaje y no cuentan con diversidad de mercados. No poseen, en síntesis, adecuada información ni acceso a créditos para formar pequeñas empresas de actividades de reciclaje; que por tal motivo se consideran factores condicionantes para garantizar una eficiencia en los niveles de productividad. Como puede verse, las condiciones en que trabajan las personas o cliente interno que hacen parte de las empresas recicladoras del plástico no son óptimas.

Por lo descrito antes, en su mayoría los recicladores del plástico no transforman el material recuperado, y por esta razón, al vender el residuo, se ven obligados a 
abandonar la fase más rentable del proceso. De acuerdo con el Servicio Nacional de Aprendizaje-SENA [2], los pequeños empresarios o personas particulares ubicados en el departamento de La Guajira desarrollan la actividad recicladora en forma desorganizada, sin definir procesos de planificación para el aprovechamiento y comercialización del plástico reciclable. Se nota la carencia de planes para gerenciar de forma eficiente y eficaz la gran cantidad de plástico que se genera en esta región colombiana.

En referencia al planteamiento realizado anteriormente sobre la eficiencia de la productividad en la actividad del reciclaje, en relación al cliente interno, es necesario predeterminar su influencia en el cliente externo, que aparece en el umbral de la institución donde el cliente interno ya ha ejecutado todas las actividades necesarias dentro de la cadena de valor de la organización, tanto en las unidades de apoyo como en las unidades operativas, y cuyo objetivo es procesar todas las tareas que agreguen valor al producto, para cumplir con las expectativas, percepción, necesidades y deseo de las empresas que se dedican a comprar el plástico reciclado, que producen y comercializan las empresas recicladoras de plástico en el departamento de La Guajira-Colombia. Para Stanton [3], "la satisfacción está enmarcada en la comparación que el cliente realiza entre el desempeño esperado y el desempeño percibido al consumir el producto".

Por tal razón, en la investigación realizada se busca conocer los niveles de satisfacción de los clientes externos, que son las empresas que compran el material reciclado, producido y comercializado por las empresas recicladoras del plástico del departamento de La Guajira-Colombia. Se debe anotar que si el desempeño del cliente interno no cumple con las expectativas, necesidades, deseo y percepción, el cliente estará insatisfecho; si las iguala estará satisfecho; pero, si las expectativas son superadas, entonces estará muy satisfecho. Al respecto, Kottler [4] afirma que "la satisfacción de necesidades se inicia con la búsqueda de necesidades reales del cliente, estimulando a este para que adquiera la mayor parte de la oferta realizadas".

Con base en la investigación, Gerencia estratégica de mercadeo como herramienta de productividad en las empresas recicladoras del plástico en el departamento de La Guajira, realizada por los autores del presente artículo, se determinó como un objetivo específico la eficiencia en la productividad desde la perspectiva del cliente interno y externo.

En este sentido, para la Corporación Regional de La Guajira [5] el proceso de producción y comercialización llevado a cabo por los recicladores de los productos resultantes del reciclaje de plástico se desarrolla de manera discontinua porque carecen de recursos necesarios para realizar en forma óptima y adecuada tal función. Muchas veces al reciclador le falta recursos financieros para comprar el material requerido que garantice una negociación satisfactoria y rentable para mantenerse en el mercado, en tanto que la mayoría de veces no tienen la capacidad para entregar a sus compradores o clientes el material requerido por ellos, es decir, no puede satisfacer las necesidades de su demanda por falta de dinero suficiente que avale un negocio rentable.

De igual forma, los recicladores carecen de tecnología adecuada para implementar el proceso de transformación del plástico [2]. Solo actúan como intermediarios para vender el material reciclado, sin poder entregar un producto transformado a un mercado carente de los mismos. Además, 
los índices de eficiencia y eficacia son inadecuados para alcanzar los objetivos y metas propuestos en estas organizaciones, razón por la que les toca vender a un precio muy bajo, que muchas veces causa el retiro o quiebra en esta actividad. La eficiencia en la productividad desde la perspectiva del cliente interno y externo, podrá generar niveles de competencia necesarios para el desarrollo organizacional y comercial, en esta empresa recicladora de plástico.

Por su parte, la Corporación Regional de La Guajira [5], en su censo de población señala que existen 32 empresas que se dedican a la compra de plástico reciclable en las ciudades de Santa Marta, Barranquilla y Cartagena, que están en condiciones de procesar más de 4.500 toneladas por día. Esto demuestra la existencia de una considerable demanda, que se puede considerar como los clientes potenciales externos de los productos resultantes del reciclaje de las empresas que se dedican a reciclar en este Departamento. Pero para lograr tal meta, se deben garantizar procesos óptimos de productividad y, de este modo, obtener una excelente percepción por parte de los demandantes.

En esta perspectiva, seguidamente se presentan: la revisión literaria; la metodología utilizada en la investigación, definiendo el enfoque epistemológico, tipo, diseño de investigación, población, muestra; así como los resultados y conclusiones del trabajo.

\section{REVISIÓN DE LITERATURA}

En el presente artículo, la revisión de la literatura se desarrolla desde dos aristas de ordenamiento bibliográfico: se realiza primero una revisión de los anteceden- tes investigativos y los fundamentos que sustentan las teorías pertinentes para determinar la eficiencia en la productividad desde la perspectiva del cliente interno y externo. En este sentido, se presentan los antecedentes investigativos, apoyándose en artículos de revistas especializadas y de investigaciones realizadas relacionadas con las variables de estudio. Los cuales se detallan a continuación:

Según el artículo, "El reciclaje del plástico Pet botella a botella tiene futuro" (2015) la empresa Aproplast procesa 1800 toneladas de este plástico por año, que rescata de los rellenos sanitarios para convertirlo en productos de alto valor. Es importante anotar que el gerente de esta empresa, Jaime Giraldo, manifiesta que todo lo recolectado se somete a un proceso técnico de clasificación, beneficio, descontaminación y limpieza, para posteriormente ser transformado, ya en otras industrias, en nuevas cajas, envases, vajillas, escobas, tejas plásticas, láminas, empaques preformados, fibras cortas y hasta juguetes. Así se realiza una importante labor social, ya que las labores de clasificación y limpieza son realizadas por una cooperativa de recicladores integrada, en su gran mayoría, por mujeres cabeza de familia. La conciencia del reciclaje que apenas despierta, se sostiene gracia al apoyo del Fondo de Modernización y Desarrollo Tecnológico de las Micro, Pequeñas y Medianas Empresas (Fomipyme).

También es de importancia el artículo, "El reciclaje: una opción para minimizar la generación de residuos sólidos urbanos domiciliarios" [6], según el cual el reciclaje es una opción para disminuir el impacto ambiental. Entre los resultados de este artículo se plantea que a pesar de que la mayoría de la población cuenta con información sobre el cuidado al medio ambiente, es mínimo el porcentaje de esta que 
realiza actividades de reciclaje desde su hogar por lo quee concluye con propuestas encaminadas a la creación de espacios y condiciones adecuadas para crear una verdadera cultura de reciclaje.

Es pertinente retomar también lo que afirma la Red de Revistas Científicas de América Latina y el Caribe-REDALYC.ORG, en su artículo titulado "El Reciclaje, La Industria del Futuro", realizado por Berenguer y otros en la Habana Cuba (2009), en el que se afirma que la industria del reciclaje es una importante suministradora de materias primas para la fabricación de los más disímiles artículos de consumo diario o de equipos de larga duración. Al mismo tiempo, protege el medio ambiente, al ahorrar los recursos minerales y la energía. Los países desarrollados son los abanderados de esta industria, que se conoce como la "Industria del Futuro", ya que cada día millones de toneladas de elementos y desechos de chatarra metálica se incorporan al proceso de reciclaje. Este artículo pone de manifiesto la importancia de realizar procesos productivos que permitan aprovechar la alta generación de residuos sólidos en el mundo en que vivimos [7].

El artículo "Logística inversa un proceso de impacto ambiental y productividad", realizado por Rodrigo Andrés Gómez Montoya y publicado por la revista Universia (2015) describe y analiza la logística inversa desde un enfoque conceptual, de procesos y aplicaciones en los niveles nacional e internacional, incluyendo la relación con la Gestión de Cadena de Suministro Verde. Además, en el artículo se observan decretos y normas que regulan la gestión de residuos en Colombia. Esta recuperación o logística inversa en algunas industrias y/o sectores se ha convertido en obligatoria para proteger el medio ambiente, y como una oportunidad para la generación de valor y beneficios económicos.

Pacheco [8] realizó una investigación titulada "Efectividad Gerencial y Productividad en las organizaciones de mantenimiento", para optar al grado de Dr. en Ciencias Gerenciales. Su objetivo fue determinar la relación entre la efectividad gerencial y la productividad en organizaciones de mantenimiento de empresas elaboradoras de cemento. Es una investigación aplicada, descriptiva y de campo, con diseño no experimental, transaccional y correlacional. En tanto que Laguna [9] realizó un estudio titulado "La Gerencia y la Productividad en los Institutos Tecnológicos Oficiales del Estado Zulia" para optar al grado de Dra. En Ciencias Gerenciales realizado en la Universidad Rafael Belloso Chacín, de Maracaibo. Esta última investigación orientó los fundamentos teóricos de la presente investigación, de igual forma ambas comparten el marco metodológico.

Por otro lado, Machado [10] realizó la investigación "Influencia del perfil gerencial y la productividad laboral del personal del sector de salud de los hospitales públicos", para optar al grado de Dr. en Ciencias Gerenciales. Se trata de una investigación descriptiva, de campo, explicativa, no experimental. Asumió como población 44 sujetos que representaron la totalidad del personal gerencial médico y de enfermeras y generó importancia como antecedente investigativo, porque la eficiencia y la productividad son variables, que serán tratadas en la industria recicladora del plástico en el departamento de La Guajira. Después de detallar los antecedentes investigativos, se presentan las teorías que sirvieron de sustento teórico como la que define la eficiencia como la capacidad para alcanzar un fin, empleando los mejores medios posibles [11]. A la vez, esta es la su- 
ficiencia de hacer las cosas en función de los resultados que se persiguen, tomando como referencia los objetivos a lograr. La eficiencia administrativa lleva a la eficiencia organizacional que se alcanza cuando se logran resultados con decisiones esenciales. En ese mismo orden de ideas, la eficiencia se asocia con los mecanismos que utiliza la organización para establecer lazos determinados en el cumplimiento de las metas, considerando el grado de satisfacción de los clientes y el manejo de los recursos.

Por su parte, Kotler [4] afirma que una empresa da eficiencia del servicio a sus clientes cuando este percibe que sus problemas son solucionados de la mejor forma posible, demostrando que la empresa tiene interés y preocupación por satisfacer sus necesidades y transmitiéndole credibilidad.. Lamb y Col [12] afirman que los empleados que tratan con respeto a los clientes y les hacen sentir que es posible confiar en una promesa son un ejemplo de confianza.

La eficiencia es un adjetivo de naturaleza cualitativa, aplicable a los procesos logísticos o a cualquier área en general, pues en condiciones ordinarias se propende a la optimización; lo que implica eficiencia, y en condiciones extraordinarias se debe cumplir la misión aún a costa de los medios, sin llegar a ser victorias pírricas, pues una alta eficiencia depende de seguir estrictamente los lineamiento de la planificación. La eficiencia es la cuantificación del cumplimiento de la meta, no importa si ésta se logra en forma eficiente o en forma efectiva.

Según Chiavenato, así como la eficiencia se determina por la cantidad de recurso utilizado en la elaboración de un producto, la tarea de la administración consiste básicamente en integrar y coordinar los recursos organizacionales, unas veces corporativos y otras veces conflictivos, tales como personas, materiales, dinero, tiempo, espacio, entre otros, para alcanzar, de la manera más eficaz y eficiente, los objetivos determinados. La eficiencia mide así el costo de los recursos asociados con el logro de una meta en función del tiempo. El costo de la mano de obra es una medida común de eficiencia, en lo que también se puede incluir el uso de equipos, el mantenimiento de las instalaciones y los rendimientos sobre el capital invertido.

En relación a la productividad, Harrinson et al [13] la definen como la relación entre la cantidad de bienes, servicios producidos, así como la cantidad de recursos utilizados, que sirve para evaluar el rendimiento de los talleres, las máquinas, los equipos de trabajo o los empleados. Es, por tanto, un sinónimo de rendimiento en el caso de los empleados En las máquinas y equipos está dada como parte de su característica técnica. Existen diferentes factores que inciden sobre la productividad de las organizaciones [11], estos factores pueden ser internos o sea que son propios de la institución y son manejados por la estructura gerenciales como: Terreno y edificio, materiales, energía máquina, equipos y recurso humano. Otros tipos de factores son los externos que no son manejados directamente por la institución, sino que dependen de condiciones externas: Disponibilidad de la materia prima, mano de obra calificada, política estatal relativa a atribuciones, aranceles, infraestructura existente, disponibilidad de capital e interés y medida de ajuste aplicada. Davis y Newstrom [14] afirman que la productividad se eleva cuando es posible generar más productos con igual cantidad de insumos o generar la misma cantidad de productos con los mismos insumos. En 
este sentido, no implica necesariamente producir más y es más bien una medida del grado de eficiencia con la que se generan los productos deseados, cualquiera que sea la cantidad de estos. La productividad es la porción de outputs (bienes y servicios) dividida por los inputs (recurso como el trabajo y el capital). Así, en el trabajo de un director de operaciones es potencial la proporción entre outputs y inputs. Por ello, mejorar la productividad significa mejorar la eficiencia, y se puede conseguir de dos formas: Reduciendo los inputs mientras los outputs permanecen constantes o aumentando los outputs mientras los inputs permanecen iguales. Las dos suponen un aumento de la productividad desde una perspectiva económica.

La productividad permite al empresario determinar la relación insumo- producto, el cual sirve para evaluar el rendimiento de los talleres, máquinas, equipo de trabajo y empleados. Al respecto Koont'z y Weihrich [1] plantean que la productividad implica eficacia tanto en el desempeño individual y organizacional. Por su parte, la eficiencia es el logro de las metas con la menor cantidad de recursos; mientras que la eficacia el cumplimiento de los objetivos.

Por todo lo expresado hasta ahora, es importante resaltar la importancia de analizar la productividad en las empresas recicladoras del plástico en el departamento de La Guajira-Colombia, con el propósito de identificar el nivel de eficiencia en el desempeño individual y organizacional de estas empresas, y así establecer el cumplimiento de sus objetivos y el logro de las metas en la utilización de sus recursos en el proceso del reciclaje llevado a cabo por las mismas, con miras a definir la relación insumos-productos pertinentes para garantizar un óptimo nivel de productivi- dad.

Desde otro punto de vista, en esta revisión literaria, conviene señalar que el cliente no es solamente quien tiene una relación comercial con la institución y hacia los cuales debe manifestarse un valor agregado perceptible, si no que existe otro, de una categoría similar o más importante para la institución, que sirve de soporte y que le ayuda a incrementar sus utilidades y a posicionar el negocio y sus productos. Desde hace unos años se han incrementado al respecto las referencias teóricas a conceptos como marketing relacional, marketing interno o cliente interno, todas ellas relacionadas entre sí. La utilización de estas nuevas construcciones conceptuales refleja un nuevo enfoque de las relaciones institución-cliente que en ocasiones no se materializa en el día a día. En concreto, la expresión cliente interno no hace sino mostrar una nueva forma de entender el proceso de productividad, en instituciones de bienes y servicios, y las relaciones que se generan dentro de la propia organización [4].

En este orden de ideas, cada empleado de la organización se convierte en un cliente interno conforme recibe su insumo, información, tarea, entre otros, de su compañero de trabajo; a su vez, él se convierte en proveedor de otro $\mathrm{u}$ otros clientes internos hasta llegar al umbral donde surgen los clientes externos, en quienes se hará realidad la calidad del producto y servicio, como reflejo de la cultura organizacional que está viviendo los clientes internos [15]. En consecuencia, cada individuo debe estar plenamente convencido de lo que recibe dentro de la institución, es decir, de las acciones que le corresponde llevar a cabo y que se dirigen a cristalizar la visión y misión, de manera que él proporcione un producto o servicio de calidad al cliente externo. 
Para Llanos en ello juega un papel clave la capacitación, que implica transmitir conocimientos, habilidades y actitudes en los empleados y trabajadores para el buen desempeño en las labores, cabal cumplimiento de las funciones en el trabajo cotidiano, y alcanzar la eficacia, eficiencia y calidad, de manera que se dignifique la actividad humana en las organizaciones.

Otro elemento importante para este fin es la motivación, que según Chiavenato es producto de factores internos o psicológicos inherentes a la persona, según las características del sujeto y las causas que provocan y motivan determinados procesos o comportamiento. Los directivos deben complementar acciones como la comunicación, compensaciones, reconocimientos, entre otras, encaminadas a incrementar la motivación, a fin de que el personal a su cargo adopte o modifique determinadas actitudes o actuaciones. Davis y Newstrom [14] destacan el compromiso, entendido como el grado en el cual un gerente o empleado se identifica con la organización y desea seguir participando en ella. Ello suele reflejar el acuerdo del empleado con la misión y metas de la organización, su disposición a empeñar su esfuerzo a favor del cumplimiento de estos, y fines de seguir trabajando ahí.

En referencia a los factores condicionantes de recursos y logística, Harrison et al [13] advierte que los recursos son los medios materiales que se pueden disponer para ser utilizado por una empresa en un determinado proceso comercial. Entretanto, Ballou considera que la logística empresarial es todo movimiento y almacenamiento que facilita el flujo de productos desde el punto de compra de los materiales hasta el punto de consumo, así como los flujos de información que se ponen en marcha, con el fin de dar los niveles adecuados de servicio al consumidor a un coste ra- zonable. Por su parte el ambiente laboral de una organización formada por seres humanos y personas empleadas en ellas es bastante subjetivo e influido por multitudes de variables, presentándose interacciones de diversas maneras, según las circunstancias y los individuos, que hacen uso de diversos factores, a su vez influidas por cuestiones internas y externas a ellos.

La importancia de los clientes internos resalta la prioridad que tienen estos en las organizaciones orientadas hacia el cliente externo. Los empleados de la institución son los mejores vendedores de sus bienes y servicios. Para ello hay que crear una adecuada cultura organizacional, lo cual requiere tiempo, constancia y dedicación. En lo que corresponde al cliente externo, Domínguez [15] refiere que está comprendido por los intermediarios que directamente tienen relación con la institución y hacia los cuales deben dirigirse las acciones estratégicas para que se manifieste un valor agregado perceptible y una calidad del producto y del servicio, que verdaderamente establezca una diferencia. Asimismo, Kotler [4] menciona que se pueden considerar dentro de esta categoría el consumidor final o usuario del servicio, quien es en última instancia es el que validará cuanto del servicio se diga o se anuncie. Hay similitud en las opiniones emitidas por los autores, pues Domínguez [15] menciona que los clientes externos están comprendidos por los intermediarios que directamente tienen relación con la institución; Kotler [4] los identifica con los consumidores finales o usuarios del servicio, y Stanton [3] los ve como el punto vital para cualquier institución.

En este sentido, conocer más a fondo las cada vez más, las necesidades, deseos, expectativa, y percepción, de los clientes externos, así como encontrar la mejor 
manera de satisfacerlas con estrategias adecuadas en una época de instituciones cambiantes, es un asunto de vital importancia para la supervivencia y prosperidad de las organizaciones. Lambín define la necesidad como un sentimiento de privación respecto a una satisfacción general, vinculada a las necesidades básicas humanas, definición que cubre la noción de necesidad genérica. Se puede suponer que existe una necesidad genérica correspondiente a cada una de las tendencias que administran la vida de los individuos; por lo tanto, no está ligada al marketing y preexiste a la demanda, ya sea en estado latente o expreso.

En lo que concierne al deseo, se puede considerar como un medio de satisfacer una necesidad. Las necesidades genéricas son estables y limitadas, los deseos son múltiples, cambiantes e influidas por la cultura. A medida que evolucionan las sociedades, mayor es el nivel de deseos de sus miembros, lo cual se traduce en demanda potencial de productos específicos cuando se acompaña de un poder adquisitivo y una voluntad de compra. Por su parte, las expectativas son esperanzas que se tienen por conseguir algo. Las expectativas de los clientes se producen por el efecto de una o más de estas cuatro situaciones: Experiencias anteriores; Opiniones de amistades, familiares, conocidos y líderes de opinión, entre otras; Promesas que ofrecen los mismos competidores; y Promesas que ofrece la misma institución [4]. Mientras que la percepción "es el proceso mediante el cual el individuo selecciona, organiza e interpreta la información que recibe para crear una imagen del mundo con significado [4]. Por último, "el proceso de recibir, organizar y dar significativos a la información o estímulos detectados por nuestros cinco sentidos recibe el nombre de percepción, desempeña un papel cen- tral en la etapa de decisión de compra" [3], en la cual se recogerían alternativas.

En este orden de ideas, destaca la importancia de analizar si las necesidades, deseos, expectativas y percepción de las empresas compradoras de plástico, ubicadas en el Caribe colombiano, actuales clientes externos potenciales de los productos resultantes de la actividad productiva y comercial de las empresas recicladoras del plástico del departamento de La Guajira-Colombia, satisfacen sus requerimientos y especificaciones, lo cual se determina en los resultados del presente trabajo, producto de la investigación Gerencia estratégica de mercadeo como herramienta de productividad en estas empresas recicladoras.

\section{METODOLOGÍA}

La investigación se llevó a cabo a través de un enfoque epistemológico positivista, que busca la forma de comprender y explicar, por tal motivo enfatiza en la actividad de la mente humana como participante activo y formativo de lo que ella conoce. Asimismo, este enfoque conduce a considerar en el análisis de la información la lógica formal como un procedimiento valido y fundamental para la consecución de la objetividad, lo que implica el uso del método inductivo, el cual se refiere a los procesos de reconocimiento que se inician mediante la observación de fenómenos, hechos o personas, con el propósito de obtener premisas y conclusiones de carácter general que puedan ser aplicadas a situaciones similares. Se instauró una postura institucionalista, cuya racionalidad está fundamentada tanto en el cientificismo como en el racionalismo, siempre que logre establecer una relación de independencia entre el sujeto que conoce y el objeto conocido. 
Esta investigación se clasificó como descriptiva, porque especificó naturalmente todas las propiedades importantes encontradas en los componentes esenciales de la eficiencia en la productividad desde la perspectiva del cliente interno y externo en las empresas recicladora del plástico en el departamento de La Guajira-Colombia. Con base a los objetivos planteados y las teorías que soportan el presente estudio, se definió también como no experimental, descriptiva, de campo y de corte transversal porque los datos fueron recolectados en un solo momento. Para tal fin se realizó la operacionalizacion de las dos variables predeterminantes en su desarrollo. En la Tabla 1 se encuentra la información pertinente.

Tabla: 1. Operacionalización de las variables Objetivo General: Analizar la Eficiencia en la productividad desde la perspectiva del cliente interno y externo en las empresas recicladoras del plástico en el departamento de La Guajira-Colombia.
La población estuvo conformada por las empresas recicladoras del plástico ubicadas en el departamento de La Guajira, que suman veinte seis (26) en total. Otra población que se tomó en consideración para el estudio fue constituido por las empresas comercializadoras o compradoras del plástico reciclable, existentes en el Caribe colombiano, las cuales son treinta y dos (32) y están ubicadas en las ciudades de Santa Marta, Barranquilla y Cartagena.

La muestra la conformó la población total de las empresas recicladoras del plástico del departamento de la Guajira, y las empresas compradoras de plástico del Caribe colombiano, porque este universo poblacional está compuesto de tan pocos integrantes que no amerita estipular muestra. Por tal motivo se realizó un censo poblacional, aplicando el instrumento al total de cada población.

Fuente: Autores.

\begin{tabular}{|c|c|c|c|}
\hline Objetivos Específicos & Variable & Dimensiones & Indicadores \\
\hline $\begin{array}{l}\text { Determinar } 1 \text { a eficiencia e } \mathrm{n} \text { la } \\
\text { productividad desde la } \mathrm{p} \text { erspectiva } \mathrm{d} \text { el } \\
\text { cliente interno en } 1 \text { as e mpresas } \\
\text { recicladoras d el p lástico en e } 1 \\
\text { Departamento de La Guajira- Colombia. }\end{array}$ & \multirow[t]{2}{*}{$\begin{array}{c}\text { Eficiencia } \\
\text { en la } \\
\text { Productividad }\end{array}$} & $\begin{array}{l}\text { Cliente } \\
\text { Interno }\end{array}$ & $\begin{array}{l}\text { Recurso y Logística: } \\
\text { Infraestructura } \\
\text { Capacidad Económica } \\
\text { Capacidad Comercial } \\
\text { Recursos Humanos } \\
\text { Materia Prima } \\
\text { Insumos } \\
\text { Equipos } \\
\text { Maquinaria } \\
\text { Condición Personal: } \\
\text { Capacitación } \\
\text { Motivación } \\
\text { Ambiente Laboral } \\
\text { Compromisos } \\
\end{array}$ \\
\hline $\begin{array}{l}\text { Identificar l a eficiencia e } \mathrm{n} \text { la } \\
\text { productividad desde la } \mathrm{p} \text { erspectiva d el } \\
\text { cliente externo en l as e mpresas } \\
\text { recicladoras d el p lástico en e } 1 \\
\text { departamento de La Guajira-Colombia. }\end{array}$ & & $\begin{array}{l}\text { Cliente } \\
\text { Externo }\end{array}$ & $\begin{array}{l}\text { Necesidades } \\
\text { Deseos } \\
\text { Expectativas } \\
\text { Percepción }\end{array}$ \\
\hline $\begin{array}{l}\text { Generar lineamientos e stratégicos para } \\
\text { potencializar la e ficiencia en } 1 \text { a } \\
\text { productividad desde la p erspectiva d el } \\
\text { cliente interno y externo en las empresas } \\
\text { recicladoras d el p lástico en e } 1 \\
\text { Departamento de La Guajira- Colombia }\end{array}$ & \multicolumn{3}{|c|}{$\begin{array}{l}\text { Lineamientos estratégicos que se diseñaran con base a } \\
\text { los resultados de los objetivos anteriores. }\end{array}$} \\
\hline
\end{tabular}


Se aplicaron dos cuestionarios: el primero fue dirigido a las empresas recicladoras del plástico en el departamento de La Guajira, constituido por sesenta y tres (63) ítems con respuestas de cinco (5) alternativas tipo frecuencia; el segundo comprendió las empresas compradoras del plástico reciclado que se encuentran en el Caribe colombiano, conformado por treinta (30) ítems dirigido a las empresas comercializadoras de plástico reciclado.

\section{RESULTADOS}

A continuación, se presentan los resultados obtenidos, con el propósito de analizar la eficiencia en la productividad desde la perspectiva del cliente interno y externo en estas empresas recicladoras. Estos resultados se originan de la tabulación, análisis, e interpretación, surgidos por la aplicación de dichos instrumentos; se fundamentan sobre las categorías de análisis de interpretación de la media, donde el
Casi siempre (4), Algunas Veces (3), Casi Nunca (2) y Nunca (1) en puntuaciones de la Media Aritmética. Estas categorías se muestran en la Tabla 2.

En relación a la dimensión cliente interno, lo concerniente a recursos y logística, se obtuvo un valor para la media aritmética de 2,58, el cual pertenece al intervalo 1,81-2,60 del Rango 2, que identifica a la categoría Bajo Nivel, con una mediana 3 y moda de 2. Esto indica que las empresas recicladoras del plástico en el departamento de la Guajira casi nunca cuentan con los recursos necesarios ni con un sistema de logística que garantice un proceso de productividad óptimo. En este orden de ideas, se puede evidenciar en la Tabla 5 , en referencia al indicador Infraestructura, que el 68,8\% de lo que respondieron el instrumento manifestaron que las empre-

Tabla 2. Categorías de análisis para la Interpretación de la media

\begin{tabular}{|l|c|c|c}
\hline \multicolumn{1}{|c|}{ ALTERNATIVAS R } & ANGO I & NTERVALO & CATEGORIA \\
\hline Siempre & 5 & $4,21-5,00$ & Muy Alto Nivel \\
\hline Casi Siempre 4 & 3 & $, 41-4,20$ & Alto Nivel \\
\hline Algunas Veces 3 & 2 & $, 61-3,40$ & Moderado Nivel \\
\hline Casi Nunca 2 & 1 & $, 81-2,60$ & Bajo Nivel \\
\hline Nunca & 1 & $1,00-1,80$ & Muy Bajo Nivel \\
\hline
\end{tabular}

rango representa la relación de la clasificación de repuestas en los cuestionarios, y para este caso, el cinco (5) representa la máxima calificación y el uno (1), la mínima.

Igualmente se diseñó una tabla que resume el comportamiento de cada dimensión, con un rango preestablecido, con sus respectivas categorías, que se calculó a partir de la longitud del intervalo existente entre los valores que se asignaron a las alternativas de respuesta, a saber: Siempre (5),

\section{Fuente: Autores.}

sas recicladoras casi nunca cuentan con la infraestructura adecuada para producir y comercializar los productos reciclados, ni desarrollan un adecuado almacenamiento para el proceso de comercialización de sus productos. En apoyo a esto, el 6,20\% respondió al respecto que nunca es así; mientras que un 18,8\% dijo que algunas veces lo era; y un 6,20\%, casi siempre. Al respecto, se observa una concordancia con la opinión de las empresas compradoras 
del plástico de la región Caribe y las empresas recicladoras, porque se comprueba que no se cuenta con la infraestructura pertinente para garantizar un buen nivel de eficiencia en la productividad.

Para el indicador Capacidad Comercial, el 39.6\% de la muestra encuestada manifiesta que las empresas recicladoras casi nunca poseen el servicio de transporte necesario para garantizar el proceso de distribución de su producto, que no tienen la capacidad comercial para ofrecer los productos cuando se requieren. Seguidamente, un $24 \%$ de los encuestados responde que nunca, mientras que un 33,3\% opinó que algunas veces era así, y un 3,10\% se inclinó por la opción de casi siempre. En dichos resultados se reconocen tendencias negativas.

Por otro lado, los resultados para el indicador Recursos humanos muestran que el $70,8 \%$ de las empresas comercializadoras o compradoras del plástico reciclable seleccionadas consideran que las empresas recicladoras casi nunca cuentan con el personal idóneo para producir y comercializar los productos reciclados, el 6,30\% respondió a esta pregunta que nunca es así; mientras que un $17,7 \%$ dijó que algunas veces lo era y un 5,20\% afirma que casi siempre. En relación a esto, se observa una moderada discordancia con la opinión de las industrias recicladoras, ya que estas seleccionan la opción algunas veces, pero igual se mantiene en la tendencia de bajo nivel.

En relación a la Materia prima, los resultados mostraron que el 68,8\% de las empresas recicladoras del plástico reconocen que casi siempre existe la materia prima necesaria para garantizar su proceso de productividad porque se genera un considerable volumen de plástico reciclable; seguidamente, un 15,6\% de los encuestados afirmó que siempre era así, mientras que un 15,6\% seleccionó la opción algunas veces. De acuerdo con lo antes dicho, se observa una concordancia con la opinión de las empresas compradoras de plástico porque afirman que en este departamento se genera una gran cantidad de este material reciclable.

Continuando con el análisis, en relación al indicador insumos, los resultados muestran que el 68,8\% de las empresas comercializadoras o compradoras del plástico reciclable encuestadas manifiestan que algunas veces las empresas recicladoras cuentan con los insumos requeridos para garantizar su proceso productivo, mientras que un 31,2\% afirma que casi nunca. Se observa al respecto una concordancia con la opinión de las industrias recicladoras porque también seleccionaron la opción de repuesta "algunas veces", aunque con tendencia positiva.

En lo concerniente al indicador equipo, el $84.4 \%$ de los encuestados considera que la empresa recicladora casi nunca posee los equipos pertinentes para reciclar el plástico, y que en su mayoría son obsoletos, afirman, además, que no tienen capacidad para adquirir equipo de alta tecnología; Un 3,10\% consideró que nunca, mientras el 9,4\% manifestó que algunas veces tienen equipos, y un 3.10\%, casi siempre. Estos resultados muestran que, sin los equipos necesarios, las empresas recicladoras no podrán ser eficientes en sus procesos de productividad. Asimismo, los resultados para el indicador maquinaria, arrojan que el 37,5\% de las empresas recicladoras del plástico casi nunca cuentan con los equipos necesarios para realizar sus procesos productivos; un 22,9\% opinó que nunca es así, 10,4\% dijo que algunas veces lo era; contrariamente a todo esto, $24,0 \%$ expresó que casi siempre, y un 15,20\%, siempre. Con base en estos resultados, se observa que estas unidades empresariales carecen 
de maquinaria para garantizar la eficiencia en sus procesos de productividad. Estos resultados se muestran en la Tabla 3.

Tabla 3. Cliente interno capacitan. Estos resultados muestran que las empresas compradoras y las empresas recicladoras reconocen que no se realiza capacitación, para llevar a cabo la productividad del plástico reciclable en el depar-

\begin{tabular}{|l|c|c|c|c|c|}
\hline \multirow{2}{*}{ Recursos y Logística } & \multicolumn{5}{c|}{ Alternativas (\%) } \\
\cline { 2 - 6 } & Siempre & $\begin{array}{c}\text { Casi } \\
\text { Siempre }\end{array}$ & $\begin{array}{c}\text { Algunas } \\
\text { Veces }\end{array}$ & $\begin{array}{c}\text { Casi } \\
\text { Nunca }\end{array}$ & Nunca \\
\hline Infraestructura & 0,00 & 6,20 & 18,8 & 68,8 & 6,20 \\
\hline Capacidad comercial & 0,00 & 3,10 & 33,3 & 39,6 & 24,0 \\
\hline Recursos humanos & 0,00 & 5,20 & 17,7 & 70,8 & 6,30 \\
\hline Materia prima & 15,6 & 68,8 & 15,6 & 0,00 & 0,00 \\
\hline Insumo & 0,00 & 0,00 & 68,8 & 31,2 & 0,00 \\
\hline Equipo & 0,00 & 3,10 & 9,40 & 84,4 & 3,10 \\
\hline Maquinaria & 5,20 & 24,0 & 10,4 & 37,5 & 22,9 \\
\hline Tendencia & $\mathbf{3 , 0}$ & $\mathbf{1 5 , 8}$ & $\mathbf{2 4 , 9}$ & $\mathbf{4 7 , 5}$ & $\mathbf{8 , 8 0}$ \\
\hline Media & $\mathbf{2 , 5 8}$ & \\
\hline Mediana & $\mathbf{3}$ & $\mathbf{2}$ \\
\hline Moda & Bajo Nivel \\
\hline Categoría & \multicolumn{7}{|c|}{} \\
\hline
\end{tabular}

Fuente: Autores.

En lo pertinente a la condición personal, en la dimensión cliente interno, se obtuvo un resultado para la media aritmética de 3,26 el cual pertenece al intervalo 2.61-3.40 del Rango 3, que identifica a la categoría nivel moderado con una mediana de 3 y una moda de 5. Esto indica que el talento humano en las empresas recicladoras del plástico del departamento de la Guajira algunas veces se preocupa por prepararse con el fin de mejorar los niveles de desempeño en los procesos de productividad; aunque estén motivados, y sientan compromiso con lo que hacen. En este sentido, se puede observar en la Tabla 6, en referencia al indicador capacitación, que el $40 \%$ de los informantes casi nunca recibe capacitación con el propósito de obtener el conocimiento necesario, que les permita mejorar el desempeño laboral; el 20.0\%, afirma, por su parte, que nunca; un $15.0 \%$ se inclina por la opción alguna veces; otro $15.0 \%$ manifiesta que casi siempre; y el restante $10 \%$ considera que siempre se tamento de la Guajira.

Para el indicador motivación, los resultados mostraron que el $40 \%$ de la muestra encuestada siempre se encuentra motivada, porque en esta zona de Colombia se genera un considerable volumen de plástico reciclable, el cual garantiza su productividad; Seguidamente, un 30\% de los encuestados prefiere la opción siempre; el 20\% marcó algunas veces; y el 10 se sitúa en casi nunca. Esta información evidencia que el personal que hace parte de las empresas recicladoras se siente motivada, factor predeterminante en la satisfacción del cliente interno. Por otro lado, los resultados para el indicador compromiso muestran que el $42.0 \%$ de las empresas recicladoras del plástico seleccionadas considera que el personal casi siempre se compromete con las tareas asumidas, porque están identificados con el quehacer diario propio de su actividad; el 35\% se identifica con siempre; el 15\% con algunas veces; y 4\% prefiere considerar nunca, y casi nunca, respectivamente. Se nota que existe compromiso por parte de los inte- 
grantes de las empresas recicladoras.

En relación al indicador ambiente laboral, los resultados mostraron que el $40.0 \%$ de las empresas recicladoras del plástico reconocen que casi nunca se da un saludable ambiente de trabajo por las condiciones locativa, carentes de requerimientos mínimos para trabajar con agrado; el 25\% manifiesta que nunca hay condiciones óptimas para trabajar; el $15.0 \%$ cree que existe un buen ambiente; y el 10\% considera que casi siempre, y algunas veces, respectivamente. Todo esto deja entrever que en este indicador hay una insatisfacción del cliente interno, lo cual influye para considerar a las condiciones personales en la categoría de nivel moderado. Los resultados referenciados con anterioridad se muestran en la Tabla 4.

Tabla 4. Cliente interno

\begin{tabular}{|l|c|c|c|c|c|}
\hline \multirow{2}{*}{ Condición Personal } & \multicolumn{5}{c|}{ Alternativas (\%) } \\
\cline { 2 - 6 } & Siempre & $\begin{array}{c}\text { Casi } \\
\text { Siempre }\end{array}$ & $\begin{array}{c}\text { Algunas } \\
\text { Veces }\end{array}$ & $\begin{array}{c}\text { Casi } \\
\text { Nunca }\end{array}$ & Nunca \\
\hline Capacitación & 10.0 & 15.0 & 15.0 & 40.0 & 20.0 \\
\hline Motivación & 40.0 & 30.0 & 20.0 & 10.0 & 0.00 \\
\hline Compromiso & 35.0 & 42.0 & 15.0 & 4.00 & 4.00 \\
\hline Ambiente Laboral & 15.0 & 10.0 & 10.0 & 40.0 & 25.0 \\
\hline \multicolumn{7}{|c|}{ Tendencia } & $\mathbf{2 5 . 0}$ & $\mathbf{2 4 . 2 5}$ & $\mathbf{1 5 . 0}$ & $\mathbf{2 3 . 5}$ & $\mathbf{2 2 . 2 5}$ \\
\hline Media & $\mathbf{3 . 2 6}$ \\
\hline Mediana & $\mathbf{3}$ \\
\hline Moda & $\mathbf{5}$ \\
\hline Categoría & \multicolumn{5}{|c|}{ Moderado Nivel } \\
\hline
\end{tabular}

Fuente: Autores.

En la dimensión cliente externo, se obtuvo un valor para la media aritmética de 2,97, el cual pertenece al intervalo 2.61-3.40 del Rango 3, que identifica a la categoría moderado nivel, con una mediana y moda de 3 . Ello sugiere que las empresas recicladoras del plástico en el departamento de la Guajira algunas veces buscan satisfacer a sus clientes externos en relación a las necesidades, deseos, expectativas y percep- ción. En este orden de ideas, en la Tabla 5 , en referencia al indicador necesidades, donde el $35 \%$ de lo que respondieron el instrumento manifestaron que algunas veces las empresas recicladoras satisfacen las necesidades de sus clientes externos, para lo cual deben ofrecer productos reciclables con los atributos y características exigidos por ellos; el $22.0 \%$ se inclina por la opción casi siempre; el $18.0 \%$ afirma que casi nunca; el $15 \%$ señala siempre y un 10\% manifiesta que nunca. Estos resultados dejan entrever que estas unidades empresariales no se preocupan por cubrir las necesidades prioritarias de las empresas compradoras del plástico en el Caribe colombiano.

Para el indicador deseo, el 32\% de la muestra encuestada manifestó que algunas veces las empresas recicladoras buscan cumplir con los deseos de las empresas 
siempre. Por tal razón, se observa que los clientes externos cubren moderadamente sus niveles de requerimientos con los productos ofrecidos por las empresas recicladoras. El indicador percepción expresa, por último, que las empresas compradoras del plástico reconocen en un $30 \%$, que algunas veces los productos ofrecidos por las empresas recicladoras los estimulan porque poseen características y atributos de buena calidad, que les permiten cubrir sus necesidades prioritarias; el 20\% afirma que casi siempre estos productos poseen las condiciones que garantizan un óptimo proceso de trasformación; el otro $20 \%$ cree que casi nunca es así.; el 18\% elige la opción siempre; y el 12\% se sitúa en la alternativa nunca. En resumen, estos resultados se establecen en la categoría moderado nivel se muestran en la Tabla 5.

Tabla 5. Cliente externo

\begin{tabular}{|c|c|c|c|c|c|}
\hline \multirow[b]{2}{*}{ Indicadores } & \multicolumn{5}{|c|}{ Alternativas (\%) } \\
\hline & Siempre & $\begin{array}{c}\text { Casi } \\
\text { Siempre }\end{array}$ & $\begin{array}{l}\text { Algunas } \\
\text { Veces }\end{array}$ & $\begin{array}{c}\text { Casi } \\
\text { Nunca }\end{array}$ & Nunca \\
\hline Necesidades & 15 & 22 & 35 & 18 & 10 \\
\hline Deseos & 11 & 17 & 32 & 18 & 22 \\
\hline Expectativas & 9 & 15 & 42 & 20 & 14 \\
\hline Percepción & 18 & 20 & 30 & 20 & 12 \\
\hline Tendencia & 13.25 & 18.5 & 34.75 & 19.0 & 14.5 \\
\hline Media & \multicolumn{5}{|c|}{2.97} \\
\hline Mediana & \multicolumn{5}{|c|}{3} \\
\hline Moda & \multirow{2}{*}{\multicolumn{5}{|c|}{$\frac{3}{\text { Moderado Nivel }}$}} \\
\hline Categoría & & & & & \\
\hline
\end{tabular}

Fuente: Autores.

\section{CONCLUSIONES}

Una vez obtenido los resultados y confrontando las bases teóricas que sustentan la presente investigación, se determinaron las conclusiones para analizar la eficiencia en la productividad desde la perspectiva del cliente interno y externo en las empresas recicladoras del plástico en el departa- mento de La Guajira-Colombia, las cuales se presentan a continuación:

Con respecto al cliente interno, en relación a recursos y logística, los resultados se sitúan en la categorías de bajo nivel, dejando entrever que se adolece de recursos y logística necesaria para desarrollar procesos de productividad con altos niveles de eficiencia; desaprovechándose, en consecuencia, la importante generación de plástico reciclable en esta zona fronteriza. Por tal razón es pertinente revisar lo que señala Harrinson et al [13], quienes mencionan que la cantidad de recursos y logística disponibles es evaluada constantemente en función de la rareza, de los cambios de usos y de las posibilidades de los elementos considerados como recursos, que se constituyen en los medios materiales disponibles en una empresa para un determinado proceso productivo y comercial; no obstante, en las empresas 
cliente realice la primera operación comercial con la organización. En lo referente a tal capacidad comercial, las empresas recicladoras no muestran capacidad para lograr el crecimiento del negocio, satisfacer a los clientes o superar la competencia de los rivales. Tampoco responden a las condiciones cambiantes del mercado, ni administran cada parte funcional del negocio para desarrollar las capacidades organizacionales necesarias para alcanzar los objetivos estratégicos y financieros.

Es menester señalar que, en estas empresas recicladoras, el recurso humano busca mejorar muy poco el desempeño del personal. Por tal razón, estas organizaciones deben administrar el recurso humano eficientemente, aprovechando los potenciales de cada persona. En relación a la materia prima, las empresas recicladoras del plástico en el departamento de la Guajira tiene la oportunidad de acceder a una suficiente cantidad de plástico reciclable porque generan 360 toneladas por día, de los cuales el $85 \%$ se está vertiendo en los rellenos sanitarios; dicha circunstancia amerita un mejor aprovechamiento con miras a la transformación de los productos reciclados, producidos por estas unidades empresariales.

En relación al indicador insumos, se puede concluir que las empresas recicladoras del plástico, , en sus procesos productivos, no aprovechan regularmente las características de los insumos en forma óptima por el desconocimiento de ciertas propiedades de calidad, y siempre buscan precios bajo, por no contar con recursos necesarios. El indicador equipo sugiere que carecen de los más necesarios para consolidar procesos productivos con altos niveles de eficiencia al igual que las maquinarias. La empresa debe diseñar políticas de manejo adecuado y preventivo para evitar riesgos laborales; aspecto que muy poco se cumple en las empresas recicladoras del plástico en el departamento de la Guajira-Colombia.

Continuando con el cliente interno, en lo que corresponde a condición personal, se concluye con base a los resultados que se encuentran en la categoría moderado nivel, mostrando que en estas empresas existe cierta preocupación por el talento humano que interactúa en los procesos de productividad, presentándose una cierta competencia de eficiencia en el personal. En lo que concierne al indicador capacitación, primeramente es importante considerar lo que afirma Llanos, quien expresa que la capacitación implica transmitir conocimientos, habilidades y actitudes en los empleados y trabajadores para el buen desempeño en las labores y un cabal cumplimiento de las funciones en el trabajo cotidiano, y de esta forma dignificar y enaltecer la actividad humana en las organizaciones. De acuerdo con los resultados obtenidos, en cuanto a este postulado teórico, las empresas recicladoras del plástico estudiadas casi nunca capacitan su personal para mejorar el desempeño en las tareas a realizar.

También se puede concluir que las personas que trabajan en las empresas recicladoras presentan altos niveles de motivación, a pesar de no contar con condiciones mínimas que garantice satisfacción permanente en las labores realizadas. Estos resultados concuerdan con los postulados teóricos propuestos por Fernández [16], quien define la motivación, como un proceso intrínseco que conduce a la persona a actuar de una determinada manera para conseguir un resultado que satisfaga sus expectativas o necesidades laborales. También se aprecia que las personas que hacen parte de estas empresas, realizan sus funciones con compromiso y dedicación, a pesar de no existir las condiciones 
laborales óptimas, para lograr la eficiencia en los procesos de productividad; en este sentido Lepeley [17] considera que conseguir un ambiente laboral equilibrado, dinámico y sin ningún tipo de alteración es una tarea difícil de conseguir.

Con respecto al cliente externo, perspectiva que debe enmarcar la eficiencia en la productividad en las empresas recicladoras del plástico en el departamento de la Guajira-Colombia, se concluye que se hallan en la categoría moderado nivel, dejando de manifiesto que las empresas compradoras del plástico reciclados no satisfacen plenamente sus necesidades, deseos, expectativas y percepción con los productos ofrecidos por las empresas recicladoras. Esto predetermina la aplicabilidad del postulado teórico del cliente externo, que según Kotler [4] se puede considerar dentro de esta categoría el consumidor final o usuario del servicio, quien es en última instancias el que validará el producto y el servicio. En referencia al indicador necesidades, se concluye que estas empresas satisfacen las necesidades de sus clientes algunas veces; por tanto, deben buscar la satisfacción de su cliente interno, con el fin de que el producto contenga las características y atributos requeridos por sus clientes externos. A la vez se reconoce una insatisfacción de las empresas compradoras del plástico reciclados, porque los productos no cumplen con los deseos prioritarios y necesarios para garantizar óptimos procesos de transformación.

En referencia a las expectativas de las empresas compradoras del plástico en la Caribe colombiano, se puede concluir que estas creen poco en las promesas realizadas por los recicladores debido a las condiciones en que estos desarrollan sus procesos de productividad; es muy cierto que las expectativas, como señalan Zeithaml y Bitner, son creencias relacionadas con la productividad, y la prestación del servicio que funcionan como estándares o puntos de referencia contra los cuales se juzga su desempeño. En este aspecto todas las conclusiones anteriores son fundamento clave por parte de las empresas compradoras del plástico para percibir el producto ofrecido por las empresas recicladoras. Estos resultados coinciden con lo afirmado por Davis [14], quien define la percepción como un proceso mediante el cual los consumidores seleccionan, organizan e interpretan los estímulos, reduciéndolos a una imagen significativa y coherente y que las personas perciben las cosas y los acontecimientos a través de todos los sentidos Finalmente cabe referirse a la generación de lineamientos estratégicos para potencializar la eficiencia en la productividad desde la perspectiva del cliente interno y externo en las empresas recicladoras del plástico en el departamento de La Guajira-Colombia, se , que se realizó con base en los resultados arrojados en los objetivos específicos, y también se apoyaron en varios aportes bibliográficos [3, 12, 13, 18]. Estos lineamientos se presentan a continuación:

- Las empresas dedicadas a la actividad del reciclaje del plástico en el departamento de La Guajira, tienen que reconocer la necesidad y deseos de los clientes actuales y potenciales, con el propósito de garantizar un nivel de satisfacción ofreciendo productos de acuerdo a las exigencias de sus clientes y así lograr un nivel de competencia estratégica.

- Para lograr la eficiencia productiva, las organizaciones recicladoras del plástico en el departamento de La Guajira deben contar, a su vez, con los siguientes requerimientos: Poseer infraestructura adecuada para garantizar su productividad; determinar fuentes de ingreso que faciliten los recursos económicos necesarios para 
realizar la actividad del reciclaje; contar con la logística necesaria para el proceso de producción y comercialización de los productos reciclados, así como con el personal idóneo y capacitado para realizar las actividades productivas y comerciales de los productos reciclados; adquirir, por último, las maquinarias y equipos pertinentes de última tecnología que faciliten el proceso de producción y comercialización de los productos reciclados.

En suma, es necesario que las empresas recicladoras del plástico en el departamento de La Guajira identifiquen y definan claramente las necesidades, deseos, expectativas y percepción de sus clientes para diseñar una estrategia de mercadeo orientada precisamente a ellos. Solo así podrán cumplir con los requerimientos de los compradores del plástico reciclado.

\section{REFERENCIAS}

[1]. H. Koontz y H. Weihrich. Administración: una perspectiva global. México: Editorial: Mc Graw Hill., 2004.

[2]. Servicio Nacional de Aprendizaje, SENA. Administración estratégica: un enfoque integrado 3a edición. Colombia: Editorial Mc Graw Hill., 2013.

[3]. [W. Stanton, M. Etzel, y B. Walker. Fundamentos de marketing. $13^{a}$ edición. México: Editorial Mc Graw Hill, 2004.

[4]. Kotler. Dirección de Marketing: La edición del Milenio. México: Pearson Educación., 2005.

[5]. Corporación regional de La Guajira, CORPOGUAJIRA, (2014), departamento de la guajira Colombia.

[6]. Y. Velázquez Narváez, (Univ. Autónoma de Tamaulipas, México). "El reciclaje: una opción para minimizar la generación de residuos sólidos urbanos domiciliarios". DELOS Desarrollo local sostenible, vol. 5 n. 15., 2012
[7]. Berenguer et al. Red de Revistas científicas de América Latina y el Caribe, REDALYC. ORG El reciclaje la industria del futuro., 2009.

[8]. Pacheco. Efectividad Gerencial y Productividad en las organizaciones de mantenimiento. Universidad Rafael Belloso Chacín, Maracaibo Venezuela., 2011. [9]. E. Laguna. "La Gerencia y la Productividad en los Institutos Tecnológicos Oficiales del Estado Zulia". Universidad Dr. Rafael Belloso Chacín. Maracaibo Venezuela., 2011.

[10]. A. Machado. Influencia del perfil gerencial y la productividad laboral del personal del sector de salud de los hospitales públicos. Universidad Rafael Belloso Chacín, Maracaibo Venezuela., 2012.

[11]. E. González. La organización social. En módulo Gerencia del Desarrollo Comunitario. Santa Fe de Bogotá: Editorial Mc Graw Hill., 2003.

[12]. CH. Lamb, J. Hair y C. McDaniel. Marketing. 6 ediciones, Thompson., 2003.

[13]. Harrinson et al. Ventaja competitiva, creación y sostenimiento de un desempeño superior compañía. México: Editorial Continental., 2003

[14]. Davis y Newstrom. Comportamiento humano en el trabajo. México. Editorial Mc Graw Hill.,2003.

[15]. Domínguez. Globalización: riesgos y realidades. Universidad Metropolitana., 2006.

[16]. Fernández. Creando organizaciones para el futuro. Chile: Dolmen ediciones., 2004.

[17]. Lepeley. Gestión y calidad en educación: Un modelo de evaluación. McGraw-Hill. Chile., 2001.

[18]. J. Gultinan y P. Gordon. Gerencia de marketing estrategias y programas. 6 edición Mc graw hill., 2004.[ 


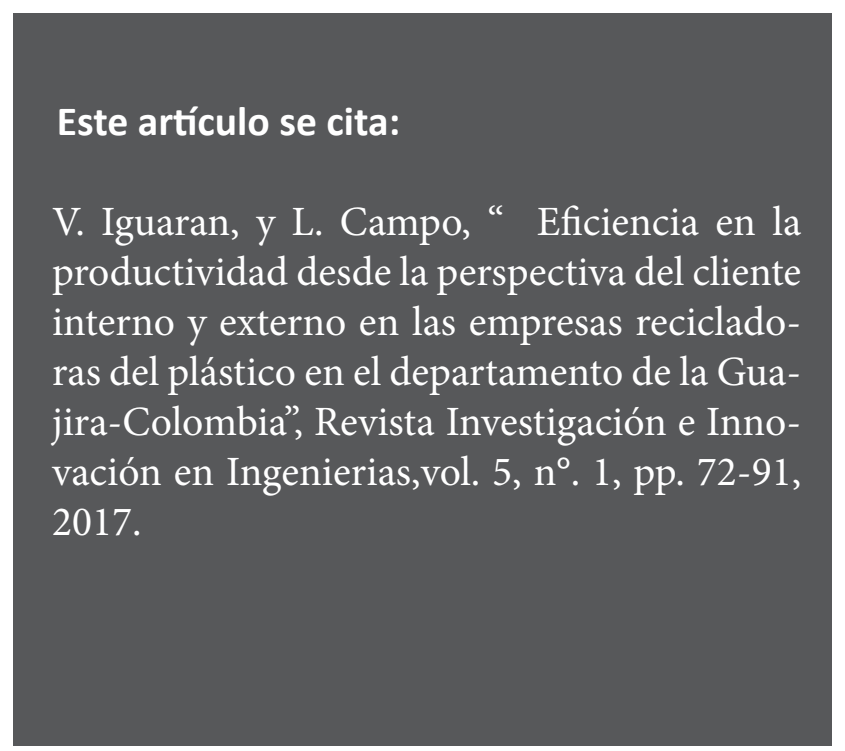

